\title{
Do electronic patient information systems improve efficiency and quality of care? An evaluation of utilisation of the Discovery HealthID application
}

\author{
S J Reid, ${ }^{1}$ BSc (Med), MB ChB, MFamMed, PhD; C Naidu, ${ }^{1}$ MSocSci; G Kantor, ${ }^{2}$ MB ChB; \\ C J Seebregts, ${ }^{3}$ BSc (Med) Hons, Dip Data, Hons BSc, PhD \\ ${ }^{1}$ Primary Health Care Directorate, Faculty of Health Sciences, University of Cape Town, South Africa \\ ${ }^{2}$ Diplomate, American Board of Anesthesiology, Department of Anaesthesiology and Perioperative Medicine, Faculty of Health Sciences, \\ University of Cape Town, South Africa \\ ${ }^{3}$ Jembi Health Systems NPC, Cape Town, South Africa; School of Public Health and Family Medicine, Faculty of Health Sciences, University of Cape \\ Town, South Africa; and Jembi Collaborating Centre for Digital Health Innovation, South African Medical Research Council, Cape Town, South Africa
}

Corresponding author: S J Reid (steve.reid@uct.ac.za)

\begin{abstract}
Background. Electronic health records (EHRs) appear to offer a number of potential benefits, but practitioners are often hesitant to make the transition to using them.

Objectives. To determine whether the use of one such system, designed and offered by a health insurer (HealthID; Discovery Health), makes a difference to the efficiency and quality of doctor-patient consultations.

Methods. A descriptive study using mixed methods was designed. A qualitative phase of individual interviews of purposefully sampled respondents was followed by a quantitative survey of a random sample of general practitioners and specialists who were registered users of the system.

Results. In the qualitative findings, 18 respondents reported their perceptions of the ease of use of the application, their motivation for using it, its functions and benefits, the impact on efficiency and quality of care, and the challenges they experienced. In addition, they reported on the details of the challenges of using the system, and made suggestions for improvements, particularly with regard to the need for training and IT support. The quantitative results from the majority of 93 respondents confirmed that while the use of the app improved patient care through positive effects on specific functions such as access to accurate patient records and easier Chronic Illness Benefit applications, they felt that it had an equivocal impact in other areas, such as maintaining patient confidentiality and enhancing teamwork and efficiency. The financial incentives offered by Discovery Health, as well as possibly the training and support provided, appear to be more influential for high-frequency than for low-frequency users. The majority said that it did not help with referrals or script writing, or with access to International Statistical Classification of Diseases and Related Health Problems, 10th revision (ICD-10) codes.

Conclusions. EHR systems like Discovery Health's HealthID could improve the efficiency of medical consultations by increasing access to stored health information without requiring data entry by clinicians, and thereby have the potential to indirectly improve the quality of care, provided that certain conditions are met.
\end{abstract}

S Afr Med J 2020;110(3):210-216. https://doi.org/10.7196/SAMJ.2020.v110i3.14111

The shift from paper-based to electronic record systems is established across all industries. In the healthcare sector, this transition offers a number of potential benefits, including access to legible, up-to-date, more complete and higher-quality information about patients; enabling better communication between clinicians and more co-ordinated care; facilitating the sharing of information with patients; supporting diagnostic processes and decision-making; decreasing prescribing errors; and reducing costs associated with storage of paper documents and with duplication of laboratory and other tests. ${ }^{[1]}$ These benefits to patients and to the system are not without limitations, however, as studies report various challenges including implementation and maintenance costs, loss of revenue associated with inefficiencies, and new kinds of medical errors. ${ }^{\left[{ }^{[2]}\right.}$ Security and privacy of electronic health records are also a concern. ${ }^{[3]}$

In the USA, the switch to electronic-based record-keeping has received significant support from policymakers espousing a national, interoperable health information system as a priority. ${ }^{[4]}$ More than $90 \%$ of all US hospitals have adopted such systems ${ }^{[5]}$ and $86 \%$ of office-based physicians had adopted an electronic health record
(EHR) by 2017.[6] Many systems fail to meet expectations, but even in these circumstances, few if any organisations return to paper and there is instead a thriving market in replacement systems from over 400 EHR vendors. ${ }^{[7]}$ Lorenzi $^{[8]}$ describes the non-technological barriers that need to be overcome to avoid system failures in introducing EHRs, and uses the behaviour change model of precontemplation, contemplation, preparation, action and maintenance to structure the stages of implementation of new electronic systems. A systematic review of barriers to EHR adoption in the USA identified cost, technical concerns and resistance to change as the major factors. ${ }^{[9]}$

In May 2012, Discovery Health introduced an electronic medical record, HealthID, to private sector doctors. The application is the first of its kind in South Africa (SA) and allows doctors, following electronically documented consent from the patient, to access certain parts of that patient's data, using a tablet computer or web interface (Fig. 1). HealthID uses data that exist in the system, so practitioners are not required to enter any new information. These data include details of previous consultations with doctors, diagnostic (International 


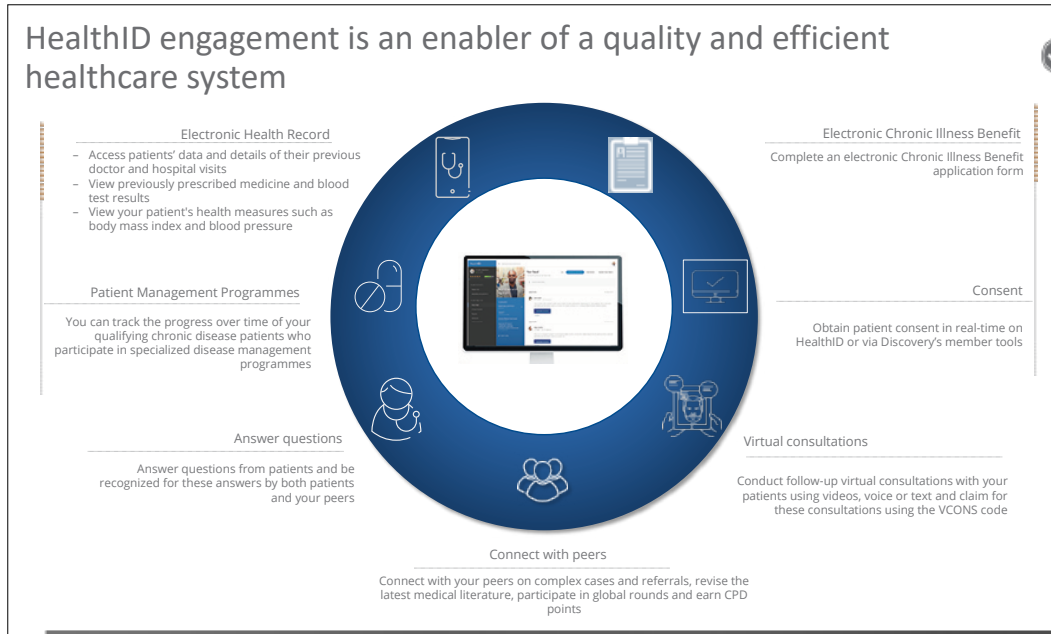

Fig. 1. Features of the HealthID application (source: Discovery Health).

Statistical Classification of Diseases and Related Health Problems, 10th revision (ICD-10)) codes and dates of interaction, hospital admissions (e.g. hospital name admission date, procedures and diagnosisrelated group), previously prescribed medicines, blood tests, sometimes with results, and patients' health measurements (e.g. body mass index, blood pressure, total cholesterol). In addition, HealthID allows for online applications for Chronic Illness Benefits (CIBs), ordering of pathology tests, scripting of medicines and referrals to other doctors. It was anticipated that this innovation would reduce the administrative burden on doctors and, by facilitating access to important patient information, improve both the quality and efficiency of care.

To facilitate adoption of HealthID, Discovery Health offered a financial incentive to doctors who utilised it beyond a threshold level over a monthly reporting period. General practitioners (GPs) who use the app receive ZAR50 per visit and specialists ZAR75 per consultation, and those who submit at least $75 \%$ of their CIB applications via the app and view the electronic record of at least $20 \%$ of their consultations are known as 'engaged' users and receive additional financial incentives.

While Discovery Health has itself internally evaluated utilisation and user experience via user surveys, focus groups and review of utilisation data, the effect and impact of this innovation have not been independently and systematically established since its launch. Almost 5000 doctors have registered and used the app at least once, and there are currently over 2500 regular users and 2000 'engaged' users as defined above, comprising both GPs and specialists (GK, personal communication, December 2019).

\section{Objectives}

The purpose of this project was to evaluate implementation of the HealthID application in SA, and there were three objectives: (i) to describe the implementation of HealthID by users and administrators; (ii) to evaluate the impact of the implementation of HealthID on the efficiency of consultations, including the utilisation of benefits; and (iii) to evaluate the impact of the implementation of HealthID on the quality of care, including the use of evidence-based medicine.

\section{Methods}

This study followed a mixed-methods approach comprising an initial qualitative phase followed by a quantitative phase. A purposeful sample was taken of HealthID users (medical practitioners), practice managers and Discovery Health Key Account Managers (KAMs), who were interviewed individually, using a semi-structured interview guide to ensure that the major issues were covered. Interviewees who were most likely to give in-depth information about the use of HealthID were purposefully chosen. Each informant invited to participate was emailed a consent form, and the interviews were conducted telephonically by an independent interviewer from a non-profit health systems company. The individual interviews lasted between 15 and 45 minutes and were recorded and transcribed with informants' consent. Medical practitioners and practice managers were given an honorarium for the time taken for the interview. The transcriptions and interviewer notes were subjected to a thematic analysis in NVivo version 9 (QSR International, Australia) to elicit major and minor themes using a deductive approach derived from the research questions, and all data were categorised according to these themes.
For the quantitative data collection, a random sample of 200 current HealthID users (GPs and specialists) was taken, estimated after consultation with a statistician, and invited to participate in the study. Results from the qualitative study were used to develop a standardised questionnaire to assess the effect of HealthID on the use of the app, the efficiency of consultations, the technical quality of care, and access to information. A high-frequency user was classified as a practitioner with at least 8 login days and 8 EHR views in a month, based on HealthID user statistics, and a low-frequency user as one with between 1 and 7 logins per month. The survey instrument was administered directly to participants by email, with online responses. Non-respondents were followed up by email twice and then phoned. Ethics approval for the study was obtained from the University of Cape Town Health Research Ethics Committee (ref. no. 273/2014).

\section{Results}

\section{Qualitative results}

Eighteen interviews were conducted, consisting of 13 respondents in practices (6 GPs, 4 specialists and 3 practice managers) and 5 Discovery Health KAMs. The key themes that were identified were ease of use, motivation, functions, benefits, impact on efficiency, and challenges. Recommendations for improvements were also made by the respondents. Verbatim responses to illustrate the major themes are given in Table 1 .

Most respondents found the Discovery HealthID app to be user-friendly and straightforward, and reported having felt 'comfortable' using it within a couple of weeks. The initial challenges pertained to understanding how to use and become comfortable with tablet/touch-screen technology and a different operating system from common desktop systems such as those based on the Windows operating system (Microsoft Corp., USA). Most respondents (both clinicians and account managers) reported that patients were generally happy to grant consent to use of the app, and in fact many were eager to contribute to improved consultations and functioning of the practice and speedier processing of patient information.

There was general agreement by clinician respondents and account managers that they were motivated to use the app by the ability to access patient records and histories for patients associated with their practices. However, these electronic records are limited to what medications they had been taking and the associated dosages, as 
Table 1. Verbatim qualitative data illustrating the major themes

Ease of use

'... there are some doctors that quite honestly had not switched on an iPad before and there are some doctors that are very tech savvy so it differs.' (KAM)

'Once he showed us, we were up and running so it is easy, I would say it is actually very user friendly and it's not really challenging, not very

much. It's actually quite easy to use it once you get into it.' (Clinician)

'They love it. They love the idea of it. $99 \%$ of the people think it's magic and magical. They think this is wonderful and everybody should do it, so they love the concept.' (KAM)

\section{Motivation for using the Discovery HealthID application}

'I actually liked the idea that you could actually access into the patient's file and you could actually see, I mean there are lots of features where you can actually see when the patient visited the doctor and those kind of things ... you can just sign in and get that information just so by the click of a button. It was a great idea, it's actually working quite nicely?' (Clinician)

... as a concept to have access to information directly, to be able to do some of the things that we need to do that we often do with forms, it's nice to able to do it online and instantly without paperwork.' (Practice manager)

'Most things are now being done electronically so that is the way forward ...' (Clinician)

'I think it varies between one of two things. They either really like the concept of the app so again you would be able to use it for chronic applications and being able to have the patient's medical history at the tip of their fingers blah, blah. Again, on the other side of the coin there are the doctors that will start using it when you start talking about the engagement fees and the extra remuneration they will get from HealthID ...' (KAM)

Training and support by Discovery Health

... go through it step by step, guide them about the device - how you do updates, how you can look for errors on the diagnostic side of it, so make sure that they are comfortable with it ... (KAM)

.... there's a couple of functionalities that I don't know how to use. I don't know if it's active yet, I was just assuming that it might not be active and for that reason I haven't used it or been trained in it.' (Clinician)

... in the first few months I didn't get paid because I didn't do a small thing. I lost a lot of money because of that, so that was not good enough and thereafter sometimes when the new system comes out, I just see that there is a new system there is no like text or something to explain to you how the new system is going to work. I work it out myself but it's an unnecessary strain to have to work it out yourself if you had a note or something just to show you how to do that.' (Clinician)

'They would train and after 2 weeks or after a month then they stopped again just to check everything - if everything was obviously going well. But, at the moment they don't follow up ... (Clinician)

Most frequently used functions

'I would say the chronic application is a big one. They do like to use that quite a bit. It's quicker, it's easier than filling in paper from a script perspective as well. Lots of them use the pathology and radiology, they do like to use that. And some of them, depending on what they specialise in, are interested in the episodes of care and the time lines that you can see on Health ID. I would say those are basically the main ones.' (KAM)

\section{Benefits of using the HealthID application}

'And then also obviously the administrative burden that it has taken away by all the paper processes that have now been replaced by HealthID, the chronic applications and the special referral for the key care plans and so on.' (KAM)

'I just log onto their profile and then I have the results so that was really nice and quick and easy.' (Clinician)

'... chronic application, once again, I've stressed that, it's been the biggest factor that's been beneficial from my side.' (Clinician)

'... I think it is actually the application that is embraced more by doctors than the actual records ... instead of filling in the paper hard copy and faxing it off and waiting for feedback and all of that.' (KAM)

'You can see on a glimpse what is going on with the patient, have they been sick, when they last saw the doctor, what they took when they went there. The patients don't always remember the details of what they took and what to remember exactly the exact duration of the illness or whatever, what investigations were done. But, just with one glance I can kind of see what is going on.' (Clinician)

'So I think it really makes their management of their patients a lot more comprehensive more like of a better word so they are able then to see exactly what type of other treatment the patient has been involved in because members don't always think about telling doctors everything.' (KAM)

'It's wonderful especially for chronic applications it's nice and quick and with different medications and like script errors which are common mistake that you make, that's a nice way of double checking that you are not prescribing the wrong dose of medication or the wrong medication. The patients often don't know what they are taking especially now that there are so many generics.' (Practice manager)

Perceived impact on efficiency/quality of care

'I don't think a dramatic impact but it facilitates maybe the history so for me it just helps with history taking so we can just get some more information from the patient. Look up the names of the medication and the previous history and things like that - that's what I find.' (Clinician) 'It just makes the admin of those things a bit easier. So I don't think it improves my quality of care, it makes my working day that bit less hasslesome in that ... I have less form filling to do. I can access information without having to get somebody or myself to phone through or find this out, etc. That's what it is.' (Clinician)

'Think it's got a very good impact ... because especially when it comes to an application to think that just by pressing a button you can actually have an answer within the same day whereas previously ... it could take anything up to 7 - 8 working days ... quite a great impact in service delivery.' (Practice manager)

Continued ... 


\section{Table 1. (continued) Verbatim qualitative data illustrating the major themes}

'All clinicians being able to make a more holistic diagnosis of their patient, by that I don't think in the past they haven't really had access to a device that has all the information of the patient in front of them so their medical history, who they have been to see before, what medication they are on, what procedures they have done, their blood results all in one application. I think it allows the doctors to make a much more educated decision on the patient's welfare.' (KAM)

Challenges in using the system

'The thing that's a bit frustrating is that it sometimes times out and sometimes it doesn't, it opens the patient profile and it hasn't opened it correctly ...' (Clinician)

'Unfortunately our system isn't as stable as it could be so there are quite a few service errors ... I think that is a big factor in stopping them from using it.' (KAM)

'... believe it or not, the most challenging is to get the patient to sign to agree to go on the system for them to sign they have to sign on the tablets and some of them are a bit skeptical, not skeptical but they obviously not set in what they are signing for and so on ...' (Practice manager)

'The fact that we haven't got paid the last few months for that ... what is annoying.' (Clinician)

'They tell us we will be able to do key care transfer of things but it hasn't materialised so it said we can look up specialists on it, that hasn't materialised.' (Clinician)

'I'm not young anymore, I didn't grow up with a computer so everything was new.' (Clinician)

'The other problem is also the reports that are supposed to be generated for the doctors, it does come from our side but there always seems to be a problem ... (KAM)

'A lot of them would like to know what their engagement statistics are because currently you only getting it like a month later than they would like to get so that is one of the systems that they would like to have put into the app where they can follow and engage how they doing on the Health ID itself. I know that is one of the priorities ...' (KAM)

\section{Changes suggested}

'They got this thing, that each time you get out of the program, you have to re-log on after a certain amount of time and that's just a bit of a pain just to log in my details after more than 20 minutes.' (Clinician)

'... if the guys are calling us from Discovery it will be nice, if we phone them and say look there is this new application and I would like to pop over and show you how to work it because now it would be useful to use.' (Clinician)

KAM = Key Account Manager.

well as records of pathology tests that had been recently performed by other clinicians or healthcare providers, rather than comprising full medical notes.

A second significant factor was that using the app was perceived to reduce the administrative burden by allowing for certain forms and other tasks to be completed online, particularly for CIB applications, which are insured benefits covering doctor visits, medications and tests for specific chronic conditions such as diabetes and hypertension.

The functions most frequently used by clinicians included access to medication history (treatment and dosage information), CIB applications, access to pathology information, and referral to specialists. There was agreement on this list of most-oftenused functions by the account managers interviewed. Clinician respondents were generally positive about the range of functions they were able to access and found it easy to navigate and to comprehend the information provided. There were, however, issues encountered relating to the referral function, which some of them reported as not always 'working as well as it should'.

According to the participants, the main benefits of using the HealthID app were that it helps to save time and reduces administrative effort, particularly with regard to ease of electronic chronic benefit applications. Many of the clinicians and account managers believed that it improved their treatment and care of patients, because they were able to access patient histories, medication use and dosage (thereby potentially avoiding possible drug interactions), and results of previous pathology tests. They found that it therefore allows for a more comprehensive approach to each patient, as they have more reliable and accurate information to work with.

Most clinician respondents did not feel that using the app necessarily improved the quality of care directly, but considered that its use in their practice would improve efficiency in various ways. Use of the app was believed to save time by having patients' medication history and dosage information easily available. The effective use of time is understood to be further enhanced owing to the reduced administrative burden related to completing forms, faxing and following up. It was also perceived that the quality of service delivery, and arguably of care, may be enhanced through the use of the app, as consultations were less focused on completing paperwork or following up on medical histories and instead allowed for improved quality of face-to-face time with patients.

The challenges faced by users of the HealthID app related to aspects such as the use of the technology on a wireless tablet, including its operating environment, patient consent, and some application functions. These difficulties were commonly raised by both groups of respondents. The challenges relating to the technical aspects, referred to as an 'unstable platform' by one account manager, included issues such as system delays and lags, being kicked out of the system, the system being 'down', inaccessible archives, and inability to access some of the functions. This was a frustration shared by both the clinician respondents and the KAMs, who were recipients of the ongoing complaints and who themselves needed to access and use the app during training and other related tasks.

Obtaining patient consent for the doctor to access the patient's HealthID record was noted to be an issue. A few patients were not comfortable granting permission, and this was sometimes believed to be due to their not wanting doctors to know that they had been for a second opinion. Although obtaining consent is time consuming, most respondents found it to be a quick and simple process and would have practice managers and receptionists discuss and obtain consent from patients prior to their consultation with the doctor.

Problems identified with the referral function were raised as a challenge. According to these respondents, the function had not been kept up to date in terms of identifying providers available for referral 
of clinicians' clients, in particular those on the Key Care programme, a medical scheme option that is more tightly managed.

Although this was not specifically a problem related to the app itself, some respondents also reported experiencing a number of general technical difficulties such as not being able to troubleshoot basic problems encountered when working on the tablet, or issues pertaining to their internet connection. These problems affected their use of the app at times, and created a sense of frustration around its use.

Clinician respondents recommended that there be more functions available on the app, for example HIV CIB application, the CIB application form, and detailed Vitality results (Vitality is Discovery's wellness programme, which offers incentives for healthy behaviours and collects health data directly from participants, including weight, blood pressure, cholesterol, smoking status and exercise data). The ability to view member funds was another frequent user request, according to the account managers. A common request by clinicians, echoed by the account managers, was that the once-off login time should be increased before the user is automatically logged off.

The need for improved support and follow-up by the Discovery Health team was also echoed by many clinician respondents. They would like to be visited regularly by their account managers and offered continuous training on new functions as well as when changes to the interface are made. One of the account managers also commented on this, with the advice that there should ideally be a dedicated Discovery representative per area to function specifically on providing technical support. One other recommendation raised by clinician respondents was that provision be made for practice managers to sign in and complete certain forms and access particular information.

\section{Quantitative results}

A total of 93 respondents completed the survey, of whom 79 were categorised as highfrequency users and the remaining 14 as low-frequency users.

Regarding use of the app, $63 \%$ responded that they were encouraged to use the app by the financial incentive, and high-frequency users were statistically more likely to likely to answer positively than low-frequency users $(p=0.002)$. In terms of training and support, less than half of the respondents (47\%) felt that the training received from Discovery Health had some effect, and $46 \%$ felt that it increased their use to some or a great extent. While $52 \%$ of high-frequency users reported greater use after training, only $21 \%$ of low-frequency users did so, but this difference was not statistically significant $(p=0.1)$. A total of $78 \%$ of respondents reported that they increased their use owing to their comfort in using iPads or other technology. These results are represented graphically in Fig. 2, with the corresponding data in Table 2 (Table 2 is available as a supplementary file at http://www.samj.org. $\mathrm{za} /$ public/sup/14111.doc).

In contrast to the qualitative data, a majority of respondents (62\%) reported that use of the app improved the quality of their care for patients, but less than half (44\%) said that its use resulted in increased face-toface time with their patients. Inversely, 30\% of respondents said that they experienced less time with their patients when using the app $(n=28)$. Less than half $(47 \%)$ felt that the use of the app improved their efficiency or patient confidentiality, and some said that the use of the app had no or only very little impact on improving teamwork among colleagues.

Most respondents (70\%) felt that using the app enabled access to more accurate patient records 'somewhat' or 'to a great extent', and over $55 \%$ found that using it enabled easy access to pathology results. While $70 \%$ of high-frequency users $(n=53)$ found that using the app enabled access to more complete records, this was experienced by only $40 \%$ of low-frequency users $(n=6)$; however, this difference was not statistically significant. Seventy-three percent of respondents found that using the app facilitated easy applications for CIBs. Fifty-six percent of respondents felt that using HealthID reduced the administrative burden of interacting with Discovery Health, while $29 \%(n=27)$ felt that it increased their administrative load. Few of the respondents
(37\%) felt that using the app facilitated access to ICD-10 codes or patient referrals, and only $17 \%$ said that it facilitated script writing. None of the $\chi^{2}$ comparisons between high-frequency and low-frequency users for any of the questions were statistically significant, apart from the effect of the financial incentive for using the app in encouraging its use.

In summary of the quantitative findings, the majority of respondents felt that while the use of the app improved patient care through positive effects on specific functions such as access to accurate patient records and easier CIB applications, it had an equivocal impact on others, such as maintaining patient confidentiality and enhancing teamwork and efficiency. The majority said that it did not help with referrals or script writing, or access to ICD-10 codes. Finally, the financial incentives offered by Discovery Health, as well as possibly the training and support provided, appeared to be more influential for high-frequency compared with lowfrequency users. These two factors appear be particularly associated with frequency of use.

\section{Discussion}

The results of this study clearly indicate some of the numerous potential benefits and pitfalls associated with the use of information and communication technology (ICT) in healthcare provision. Research on the adoption of EHR systems in developed countries has shown associations with improvements in health equity and care to the under-served, enhanced patient safety, greater co-ordination of care through electronic sharing, and improved adherence to evidence-based healthcare through digital clinical support. ${ }^{[10]}$ The respondents in this study primarily reported on benefits relating to access to more accurate and reliable

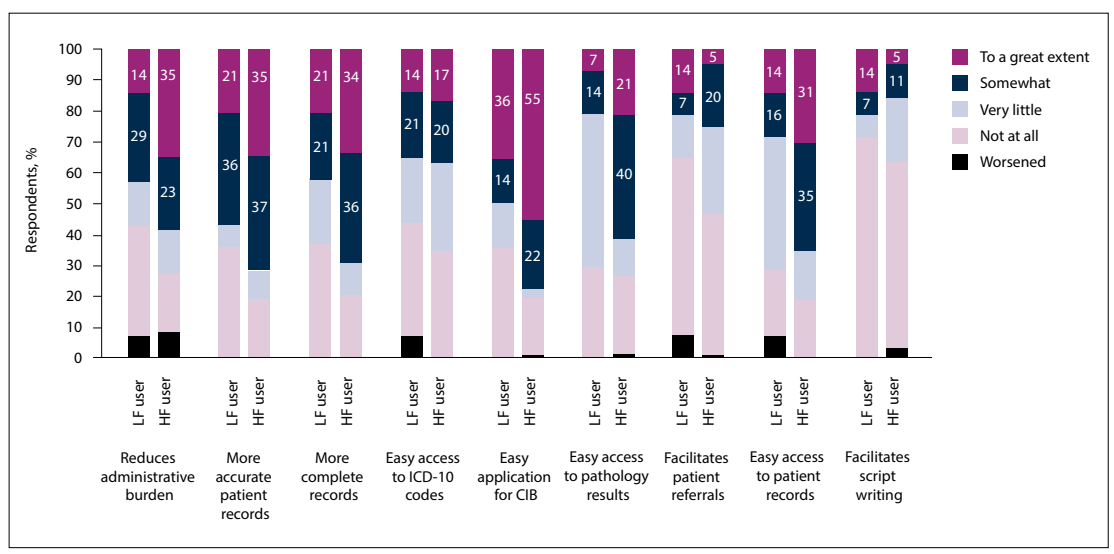

Fig. 2. Extent to which the HealthID application improved administrative aspects of respondents practice. $(L F=$ low-frequency; $H F=$ high-frequency; ICD-10 = International Statistical Classification of Diseases and Related Health Problems, 10th revision; CIB = Chronic Illness Benefits.) 
information, which may have an impact on administrative efficiency, and indirectly on the quality of care provided to patients. These results are supported by research by Jamoom et al., ${ }^{[11]}$ who reported on a national sample of clinicians' perceptions of EHRs. They found that $79 \%$ of providers felt that their practice functioned more efficiently and $82 \%$ reported that time was saved through sending prescriptions electronically. With time being a critical factor in a human resource-constrained and overburdened public health system in SA, the benefits of efficiency shown in the present study would be likely to apply in the public sector with a similar kind of app. As the Discovery HealthID app is only being used by a proportion of doctors in the private sector, the potential benefits or challenges that would be experienced by users in the public sector may be different.

Baron et al. ${ }^{[12]}$ state that five broad issues that promote successful implementation of EHRs are 'financing; interoperability, standardization, and connectivity of clinical information systems; help with redesign of work flow; technical support and training; and help with change management'. While the most useful features for users were access to medication history and pathology information, CIB applications, and support for referral to specialists, the HealthID app has many other potentially useful features that have not been as well received or utilised. The lack of perceived assistance with script writing in this study, for example, may be due to inadequate training or uptake, since the HealthID app is equipped with this function. This finding speaks to the need for further training and development of users of the application, to ensure that the potential advantages are optimised. A common recommendation made by the study respondents, both clinicians and Discovery Health KAMs, was for more training. The literature also confirms that more capable and fully functional systems offer the potential for greater benefits to users. ${ }^{[5]}$

The uptake and use of the app by different groups of users was reflected in the results of the present study, particularly the difficulty in persuading low-frequency users both to start using the app and to respond to the survey. This result follows the pattern of so-called 'early adopters' and the 'late majority' with respect to new technology originally described for farming practices in the $1960 \mathrm{~s}^{[13]}$ It is notable that the financial incentive for the use of the app was the only statistically significant difference between high-frequency and low-frequency users, even though the numbers of participants in this study was small. It is possible that some stimulus, financial or other, is needed to encourage potential users to try out the app and start using it. ${ }^{[14]}$ The need for incentives indicates a broader issue regarding the initial motivation to start using EHRs at all, which is an important area for further research.

These results also raise the important factor of the technical support that is needed for institutions, particularly those that are already under-resourced and lacking staff with the necessary expertise to navigate and trouble-shoot ICT systems, which is particularly relevant in the SA context. ${ }^{[15]}$ These concerns are also raised by the World Health Organization, ${ }^{[16,17]}$ which cites obstacles to the introduction and optimal use of automated health records as including limited availability of technology, lack of technical expertise and computer skills of staff, and lack of data-processing facilities. These challenges were referred to by users in this study as an 'unstable platform' and included issues such as system delays and lags, the system being 'down', and inability to access some of the functions. Encountering technical problems of this nature not only creates frustration and demotivates future use of the app but also contradicts a critical claimed benefit, which is to save time and improve workflow. The literature also notes this potential disadvantage of productivity losses and disruption of workflow related to the learning and technical difficulties associated with EHRs, which may potentially lead to revenue losses in the short and long term. ${ }^{[1]}$

\section{Study limitations}

Limitations of this study include self-reported data that cannot be independently verified, and, as a small sample of users were interviewed and surveyed, a large potential margin of error in the reported results, including user satisfaction and user estimates of the effect of the application. Remuneration of respondents for their time to participate in the research may have introduced a bias to the results. Finally, despite repeated efforts to recruit low-frequency users of the app to participate in the survey, a satisfactory response rate was not achieved and the quantitative comparison of high- and lowfrequency users was compromised. The results are likely to be skewed towards those with greater receptivity to the use of the app, but this is mitigated by random sampling and the methodological triangulation of qualitative and quantitative sources of data.

\section{Conclusions}

This study provides insight into the key benefits and challenges experienced by a sample of medical practitioners, both GPs and specialists, who use the Discovery HealthID application on a relatively frequent basis. While respondents reported many advantages, they also articulated the challenges that exist. We conclude that EHR systems such as HealthID could improve the efficiency of medical consultations by increasing access to stored health information without requiring health data entry by clinicians, and thereby have the potential to indirectly improve the quality of care, provided that certain conditions are met. These conditions include an incentive or other motivation to start using EHRs in the first place, a reliable digital system, and adequate training and support. The findings obtained suggest that, with the necessary infrastructure and technical support in place, an EHR system promises improved workflow efficiency that could translate to cost savings as well as improved quality of care. The lessons learned may therefore be informative for other healthcare providers as well as national government in their pursuit of an electronic system of record-keeping and medical consultation, which should contribute to achieving universal health coverage. ${ }^{[10]}$ Key to its success is that substantial investments are needed to ensure that practitioners are adequately prepared, that the electronic system is reliable and interoperable, and that financial and information technology support is available.

\section{Declaration. None.}

Acknowledgements. The authors acknowledge the work of Rhonwyn Cornell from Jembi Health Systems, who carried out the interviews and compiled the data.

Author contributions. SJR, CJS and GK conceptualised the study, CN collected and prepared the data, and all authors analysed the results. SJR drafted the manuscript and all authors contributed to the final version.

Funding. This project was funded by a research grant from Discovery Health. Conflicts of interest. Particular care was taken by the research team based at the University of Cape Town and Jembi Health Systems to sample respondents and collect and analyse the data independent of Discovery Health as far as possible. While GK was a paid consultant to Discovery Health at the time of the study and therefore understood the system, he was not involved in collecting or analysing the study data. At the time of submission, $\mathrm{CN}$ was employed by the journal/publishers to which the manuscript was submitted. 
1. Menachemi N, Collum TH. Benefits and drawbacks of electronic health record systems. Risk Manag Health Policy 2011;4:47-55. https://doi.org/10.2147/rmhp.s12985

entrom Heath Health Finance Manager 2002;56(1):52-57. h/ps.//Ww.hebi.nlm.nih.gov/pubmed/11806319 (accessed 8 March 2019).

3. Fernández-Alemán JL, Señor IC, Lozoya PAO, Toval A. Security and privacy in electronic health records: A systematic literature review. J Biomed Inform 2013;46(3):541-562. https://doi.org/10.1016/j. jbi.2012.12.003

4. Jha AK, DesRoches CM, Campbell EG, et al. Use of electronic health records in U.S. hospitals. N Engl J Med 2009;360:1628-1638. https://doi.org/10.1056/nejmsa0900592

5. DesRoches CM, Charles D, Furukawa MF, et al. Adoption of electronic health records grows rapidly, but fewer than half of US hospitals had at least a basic system in 2012. Health Affairs 2013;32(8). https://doi.org/10.1377/hlthaff.2013.0308

6. National Coordinator for Health Information Technology. Health IT dashboard. https://dashboard. healthit.gov/index.php (accessed 8 March 2019).

7. Black Book Rankings. Top Ambulatory Electronic health records vendors: Primary care physicians, 2017. http://www.practicefusion.com/brown-wilson-black-book-rankings/ (accessed 28 June 2019).

8. Lorenzi NM. Beyond the gadgets. BMJ 2004;328:1146-1147. https://doi.org/10.1136/bmj.328.7449.1146

9. Kruse CS, Kristof C, Jones B, et al. Barriers to electronic health record adoption: A systematic literature review. J Med Syst 2016;40:252. https://doi.org/10.1007/s10916-016-0628-9

10. Jha AK, DesRoches CM, Kralovec PD, Joshi MS. A progress report on electronic health records in U.S. hospitals. Health Affairs 2010;29(10):1951-1957. https://doi.org/10.1377/hlthaff.2010.0502
11. Jamoom E, Patel V, King J, Furukawa M. EHR adopters vs. non-adopters: Impacts of, barriers to and federal initiatives for EHR adoption. Healthcare 2012;2(1):33-39. https://doi.org/10.1016/j. si.2013.12.004

12. Baron RJ, Fabens EL, Schiffman M, Wolf E. Electronic health records: Just around the corner? O over the cliff? Ann Intern Med 2005;143(3):222-226. https://doi.org/10.7326/0003-4819-143-3over the cliff?

13. Rogers EM. Diffusion of Innovations. 1st ed. New York: Free Press of Glencoe, 1962.

14. Bower AG. The Diffusion and Value of Healthcare Information Technology. 2nd ed. Santa Monica, Calif.: Rand Corporation, 2005.

5. Yogeswaran P, Wright G. EHR implementation in South Africa: How do we get it right? Stud Health Technol Inform 2010;160:396-400. https://doi.org/10.3233/978-1-60750-588-4-396

16. World Health Organization. Electronic Health Records: Manual for Developing Countries. Manila Western Pacific Regional Publications, Philippines, 2006. http://www.wpro.who.int/publications/docs/ EHRmanual.pdf (accessed 28 June 2019).

17. World Health Organization. Global diffusion of eHealth: Making universal health coverage achievable Report of the third global survey on eHealth. Global Observatory for eHealth. 2016. http://apps.who. int/iris/bitstream/10665/252529/1/9789241511780-eng.pdf (accessed 28 June 2019).

Accepted 16 August 2019 